\title{
Comparison of genetic services with and without genetic registers: access and attitudes to genetic counselling services among relatives of genetic clinic patients
}

\author{
L Kerzin-Storrar, C Wright, P R Williamson, A Fryer, A Niindou, O Quarrell, D Donnai, \\ D Craufurd
}

J Med Genet 2002;39:e85(http://www.jmedgenet.com/cgi/content/full/39/12/e85)

See end of article for authors' affiliations

Correspondence to: MS L Kerzin-Storrar Academic Group of Medical Genetics and Regional Genetic Service, St Mary's Hospital, Hathersage Road, Manchester M13 OJH, UK: Lauren.Kerzin-Storrar@cmmc. nhs.uk

\begin{abstract}
The pedigrees of 192 subjects at risk of Duchenne or Becker muscular dystrophy, myotonic dystrophy, or balanced chromosome translocations attending three regional genetic clinics were inspected to identify relatives who were themselves at high risk of these disorders. Of the 342 relatives eligible for inclusion, $43 \%(63 / 147)$ of the register relatives and $26 \%(50 / 195)$ of the non-register relatives had had contact with the clinical genetic services, a significant difference $(p<0.02)$. Relatives from families with muscular dystrophy were significantly more likely to have been in contact with genetic services than those from BT families. Fifty-two relatives were interviewed about their experience and attitudes regarding genetic counselling. Almost all regarded knowledge about the family genetic disorder as helpful, and only one thought it unacceptable for relatives to be informed that they are at risk; $94 \%$ thought it was acceptable for this information to come from family members, $92 \%$ from general practitioners, and $90 \%$ from the clinical genetic service. A majority of relatives (53\%) thought it was the family's responsibility to pass on genetic risk information, but $22 \%$ said the genetic service should be responsible and $18 \%$ thought it should be the GP. These data, together with the findings from the study of probands attending genetic clinics for these disorders, indicate that the genetic register approach incorporating long term follow up and a proactive approach to genetic counselling is acceptable to the families concerned and improves access to genetic services for at risk relatives.
\end{abstract}

I the preceding paper we reported the results of a study evaluating the genetic register service in a sample of 192 people referred for genetic counselling because of a family history of Duchenne (DMD) or Becker (BMD) muscular dystrophy, myotonic dystrophy (MD), or a balanced chromosome translocation (BT). The study compared subjects attending one of two centres offering a conventional clinical genetic service with those attending a third centre, which has instituted a genetic register service for families with these four disorders. The main features of the register service are a system of annual follow up for subjects who chose to be included on the register, and a proactive approach towards offering genetic counselling to other at risk family members. One of the primary aims of this study was to elicit the views and opinions of family members regarding the acceptability of this proactive approach to informing other relatives of their at risk status.

Interviews with the probands showed that $98 \%$ thought it acceptable for relatives to be informed of their at risk status by another family member, and $78 \%$ had no objection to this information coming directly from the clinical genetic service. However, all of these subjects had been previously seen in the genetic counselling clinics, and it is therefore possible that their views may not have been representative of family members as a whole. In order to overcome this potential problem, we report here on the experiences and attitudes of at risk relatives identified from the pedigrees of the probands interviewed in the first part of this study.

\section{METHODS}

\section{Selection of subjects}

The overall study design has been explained in the previous paper (ref proband paper). The names of all potentially at risk relatives of the study probands were ascertained from the pedigree recorded in the notes at the three participating genetic centres. Relatives were considered eligible for inclusion in the study if they were aged 18-55 in December 1996, had a prior genetic risk of $10 \%$ or greater of being either a carrier or affected by the relevant condition, and were still living in the geographical catchment area of the genetic centre where the proband had been seen. The case records in the three genetic centres were then inspected to determine whether each eligible relative had had any previous contact with the genetic service.

It was decided to interview one relative from each family, to ensure that the views expressed were independent. All the eligible relatives were identified and one was randomly selected; the proband was then asked to provide a current address and permission to contact the chosen relative by post. If the proband refused permission to approach the selected relative, or the relative declined to participate in the study, another relative was randomly selected and the whole process repeated until one relative had agreed to take part or no further eligible relatives were available.

\section{Data collection}

Subjects were interviewed in their own homes, using a semistructured interview schedule similar to that used to collect data from the probands (ref proband paper). They were also asked to complete the same standardised, self-administered questionnaires. The relatives' semi-structured interview included additional questions for those who had not had genetic counselling, exploring the reasons why they had not been seen and their perceptions about access to the genetic counselling service. The interviews were tape recorded and subsequently transcribed for analysis.

\section{Analysis}

Relatives who had previously received genetic counselling were compared with those who had not. For binary outcomes, the two groups were compared using Fisher's exact test. For 
Table 1 Characteristics of 52 interviewed relatives, comparing those who had previously received genetic counselling with those who had not

\begin{tabular}{|c|c|c|}
\hline & $\begin{array}{l}\text { Previous genetic } \\
\text { counselling }(n=29)\end{array}$ & $\begin{array}{l}\text { No previous genetic } \\
\text { counselling }(n=23)\end{array}$ \\
\hline \multicolumn{3}{|l|}{ Site } \\
\hline Manchester & $15(52 \%)$ & $5(22 \%)$ \\
\hline Liverpool & $9(31 \%)$ & $10(43 \%)$ \\
\hline Sheffield & $5(17 \%)$ & $8(35 \%)$ \\
\hline Sex: Female & $24(83 \%)$ & $15(65 \%)$ \\
\hline Age: mean (range) & 35 (18-46) & 36 (22-49) \\
\hline \multicolumn{3}{|l|}{ Condition } \\
\hline $\mathrm{BMD}$ & $4(14 \%)$ & $2(9 \%)$ \\
\hline DMD & $7(24 \%)$ & $3(13 \%)$ \\
\hline$M D$ & $10(34 \%)$ & $2(9 \%)$ \\
\hline BT & $8(28 \%)$ & $16(70 \%)^{*}$ \\
\hline \multicolumn{3}{|c|}{ At least one affected (BMD/DMD/MD) or carrier (BT) first degree relative } \\
\hline BMD & $2(50 \%)$ & $1(50 \%)$ \\
\hline DMD & $2(29 \%)$ & $0(0 \%)$ \\
\hline$M D$ & $9(90 \%)$ & $1(50 \%)$ \\
\hline BT & $3(38 \%)$ & $6(38 \%)$ \\
\hline Modified Lerman worry score: mean (SE) & $4.6(0.61)$ & $3.3(0.52)$ \\
\hline STAl state anxiety score: mean (SE) & $35.6(2.2)$ & $32.1(2.9)$ \\
\hline
\end{tabular}

Table 2 Relatives' awareness of being at risk, and knowledge of the actual genetic risk for themselves and their children: comparison of those who had previously received genetic counselling with those who had not

\begin{tabular}{lll}
\hline & $\begin{array}{l}\text { Previous genetic } \\
\text { counselling (n=29) }\end{array}$ & $\begin{array}{l}\text { No previous genetic } \\
\text { counselling (n=23) }\end{array}$ \\
\hline $\begin{array}{l}\text { Previously aware of condition } \\
\text { Yes }\end{array}$ & $24(83 \%)$ & $17(64 \%)$ \\
No & 5 & 6 \\
How found out about condition & 1 & 0 \\
Birth/diagnosis of affected child & 3 & 0 \\
Own diagnosis & 19 & 17 \\
Information from relative & 1 & 0 \\
Other & 10 & 7 \\
How found out about genetic risk & 0 & 0 \\
At same time as above & 5 & 2 \\
While pregnant & 6 & 0 \\
Own diagnosis & 2 & 2 \\
Genetic counselling & 1 & 6 \\
Other & & $8(35 \%)$ \\
Missing & $14(50 \%)$ & $7(30 \%)$ \\
Knowledge & $11(39 \%)$ & $4(19 \%)^{*}$ \\
Correct risk for self & $9(50 \%)$ & \\
Correct risk of child being affected & & \\
Correct risk of child being carrier & & \\
(DMD/BMD and BT only) & &
\end{tabular}

continuous outcomes where the assumption of normality seemed appropriate, a two sample $t$ test was used. Open questions were analysed using qualitative methods to identify themes and categories. ${ }^{1}$ Coding categories were agreed by two separate researchers. Some open questions were then entered on the database with the quantitative data and analysed in the same way.

\section{RESULTS}

\section{Characteristics of population}

Of 192 probands interviewed in the first part of the study, 66 $(34 \%)$ did not have a relative in the eligible age range and living in the genetic service catchment area. The remaining 126 (66\%) probands had a total of 342 eligible relatives between them. Fourteen of the 126 probands with eligible relatives refused permission for any relative to be contacted; reasons given for doing so included a lack of discussion in the family, the wish to protect relatives from distress, and probands' perceptions that the relative would not wish to participate. The remaining 112 probands gave permission for at least one relative to be contacted, and 154 relatives were approached, of whom 52 agreed to be interviewed. The response rate in the relatives who were actually approached was therefore 33\% (52/154). Most of the relatives interviewed were first degree relatives of the proband $(43 / 52,83 \%)$, and more than half reported having at least weekly contact with the proband $(27 / 52,52 \%)$.

The demographic characteristics of the relatives who agreed to be interviewed and those who were approached but not interviewed were examined for evidence of a systematic response bias. There were no significant differences between the two groups with respect to condition, age, gender, or site. Twenty-nine $(56 \%)$ of the relatives interviewed reported a previous contact with the clinical genetic service, compared with 70 $(48 \%)$ of those who were approached but not interviewed.

\section{Access to genetic counselling}

Overall, $30 \%$ of eligible relatives, $48 \%$ of relatives approached, and $56 \%$ of interviewed relatives had a record of previous contact with the genetic service. As expected, a significantly larger 
Table 3 Relatives' opinions concerning the benefits of knowing about their own genetic condition, the acceptability of informing other family members about their at risk status, and whose responsibility it should be to do so

\begin{tabular}{lc}
\hline & Total \\
\hline Benefits of knowing about their own genetic condition $(\mathrm{n}=48)$ & $46(96 \%)$ \\
Knowledge had been helpful & $1(2 \%)$ \\
Would have preferred not to know & $1(2 \%)$ \\
Not sure & $48(94 \%)$ \\
Acceptability of informing other family members about genetic risk $(\mathrm{n}=51)$ & $46(90 \%)$ \\
Family member & $47(92 \%)$ \\
Genetic service & $0(0 \%)$ \\
GP & 0 \\
Not acceptable at all & $27(53 \%)$ \\
Responsibility for informing family members about genetic risk $(\mathrm{n}=51)$ & $11(22 \%)$ \\
Not acceptable & $9(18 \%)$ \\
Family member & $2(4 \%)$ \\
Genetic service & $2(4 \%)$ \\
GP &
\end{tabular}

proportion of eligible relatives had received genetic counselling if they lived in the catchment area of the centre with a genetic register service (register: 63/147, 43\%; non-register: 50/195, 26\%; $\mathrm{p}<0.001)$.

Among the 52 relatives who were interviewed, those who had previously received genetic counselling did not differ significantly from those who had not in terms of age, sex, or personal experience of the family condition (table 1), although there was a trend for the counselled group to contain a higher proportion of females. As all relatives had to have a prior risk of $10 \%$ or greater to be eligible for the study, this trend is unlikely to be explained by a difference in sex related risks for some of the conditions included in the study. However, only eight $(28 \%)$ of the previously counselled relatives came from families with a chromosome translocation, compared with 16 $(70 \%)$ of the relatives who had not received counselling. Relatives from BMD, DMD, and MD families accounted for 21 $(72 \%)$ of the previously counselled group, but only seven $(31 \%)$ of the non-counselled group, a statistically significant difference $(\mathrm{p}<0.02)$.

Although there was documentary evidence in the medical records that $29(56 \%)$ of the 52 interviewed relatives had received genetic counselling, five of these 29 denied that they had ever had such an appointment! Twelve of the 24 relatives who recalled having had genetic counselling had been contacted directly by the genetics service, four had contacted the genetic service themselves at the suggestion of a relative, two had been referred by their GP, two had been referred by a hospital specialist, three attended the appointment with the proband, and one was unable to remember how the appointment had been arranged. However, 19 of the 24 said that they had first learned of their family history from another family member, and only six had found out they were at risk themselves as a result of genetic counselling (table 2).

Of the 23 relatives who had not had genetic counselling before the study, 10 (43\%) said they had heard of the genetics service. Six $(26 \%)$ said they were previously unaware of a possible genetic risk. Only six (26\%) said it had ever been personally suggested to them that they could have a genetic counselling appointment; of these, the suggestion was made by a family member in five cases, and directly by the genetic service in one. Fourteen of the 23 were able to give further insight into why they had not accessed genetic counselling. Several felt they had not had sufficient information to access the service: "Well I would have liked them to get in touch from, you know from finding out there is a problem. Whereas at the moment it's been relied on family members just passing it down, and we're all ignorant, we don't know what it is, you know what it involves and so on." [Sheffield BT relative]

Others did not perceive that they were at significant risk or that genetic counselling would be relevant to them at the time: "I've always known [about genetic condition] but it's been more prominent lately obviously because I'm living with someone. I do want to get married and have a baby next year, so it's more of an immediate problem now. It was always something I knew but it didn't really matter to me at the time." [Liverpool DMD relative]

In one case the relative had been told by the proband (incorrectly) that they would not be at risk, and two relatives described reluctance on their own or their relative's part: "Fear I think, yes just a reluctance to pursue things. I think because I'd already had my children, but I wasn't far seeing enough about it really because I've had two daughters so they may well be carriers...I think I actually did want to know but actually my two daughters were quite reluctant also. So I just held back on their behalf really." [Liverpool BT relative]

Ten $(43 \%)$ of the 23 relatives interviewed who had not had genetic counselling requested an appointment following the research interview. This included four people who had previously been offered genetic counselling.

\section{Knowledge of genetic risks}

Fewer than a half of the relatives interviewed were able correctly to identify the correct genetic risk category for themselves $(42 \%)$ and their children (risk of being affected $35 \%$, risk of being a carrier $25 \%$ ). There was no significant difference in knowledge between the previously counselled and uncounselled groups, although there were trends towards more accurate knowledge in the counselled group (table 2).

\section{Impact and adjustment}

The 12 relatives who had been offered genetic counselling through a direct contact made by the genetic service were asked if they could recall their feelings at the time of the initial contact. The interview began with an open ended question, and the subject was then prompted to agree or disagree with each of the following emotions: surprised/shocked, anxious/worried, angry/annoyed, pleased/relieved. Two of the 12 could not recall any emotional response to the offer of a genetic counselling appointment. None admitted to feelings of anger or annoyance, and just two said they felt surprised or shocked. Nine relatives remembered feelings of relief. Many described mixed emotions: six remembered feelings of anxiety and apprehension but five of these six also described feeling 
relieved and/or pleased that the contact had been made: "Relieved I think on a personal level because I didn't really understand what was going on, and it was somebody to clarify." [Manchester DMD relative, anxious and pleased] "Nothing really, just ...... wanted to know what was going on, I was glad really." [Manchester BMD relative, anxious, pleased, and relieved]

Asked whether knowing about the genetic condition in their family had helped, all but two of the 48 relatives who replied to this question thought that the knowledge had been helpful (table 3), and there was only one person who would definitely have preferred not to know: "I would have preferred not to know, actually. I always said that all along but, because I had her (daughter) and she's fine, I had me little lad and he's fine, and I've never had miscarriages, so really I would never have known that I was a carrier" [Liverpool BT relative]

In the group as a whole, there were no significant differences between those who had previously received genetic counselling and those who had not with respect to scores on the modified Worry scale or the STAI.

\section{Acceptability and responsibility for informing relatives} None of the relatives interviewed said that it was unacceptable for relatives to be proactively informed about their genetic risk. Most people thought it was acceptable for either the family $(48 / 51,94 \%)$, the GP $(47 / 51,92 \%)$, or the genetic department $(46 / 51,90 \%)$ to contact relatives. When asked who should have the main responsibility for passing on this information, just over half the relatives thought it was the family's responsibility $(27 / 51,53 \%)$, while $11(22 \%)$ thought the genetic department should assume responsibility and nine (18\%) thought it was the GP's responsibility to inform relatives of their genetic risk. There was a trend for those who had previously had genetic counselling to think it the genetic department's responsibility (previous genetic counselling $10 / 29,35 \%$; no genetic counselling 3/22, $14 \%$ ) rather than that of the family alone (genetic counselling 13/29, 45\%; no genetic counselling 14/22, 64\%).

\section{DISCUSSION}

\section{Recruitment}

The intention at the outset of the study was to recruit one relative for every proband, in order to compare the experiences of relatives from the centre operating a genetic register service with those from the two more traditional genetic centres. There are several reasons why this aim could not be achieved. One third of the probands did not have an eligible relative living in the geographical catchment area of the study, and of those who did, 9\% were unwilling to allow access to any of their relatives. Some of the probands with one or more eligible relatives who did consent to this part of the study were nevertheless selective about which relatives they would allow us to contact. The response rate of $33 \%$ in the relatives who were approached was also considerably lower than among the probands, although this was expected in view of the fact that all the probands had previously been seen in one of the three genetic centres. It is therefore possible that those relatives who did take part in the study were not truly representative of the overall population of at risk family members. However, a comparison of relatives who were interviewed with those who were approached but declined found no systematic differences in demographic characteristics such as age, sex, or family condition.

\section{Access to genetic counselling}

Among the 342 eligible relatives, significantly more from the catchment area of the centre operating a genetic register service had previously received genetic counselling than those from the areas without a register. This is evidence that the register service does succeed in improving access to genetic counselling services. There was also a difference between the relatives at risk of BT and those with a family history of BMD, DMD, or MD; significantly more relatives of the muscular dystrophy probands than of the BT probands had been seen for genetic counselling. This difference may reflect a greater emphasis on extending the offer of genetic counselling to at risk members of muscular dystrophy families in all three centres.

Most of the relatives had learned of their at risk status from another family member before any contact with the clinical genetic service, and even among those who had not previously been seen for genetic counselling, there were very few who were completely unaware of their at risk status. Some of the relatives who had not attended genetic clinics were aware from discussions with other family members that the genetic counselling service was available, but had chosen not to pursue this because of anxiety or ambivalence, or because they had not yet reached a stage in life where issues such as reproductive decision making would make genetic counselling relevant to their needs. However, several relatives indicated that they would have liked to access the genetic service but did not have sufficient information to do so. It was noteworthy that almost half of the group who had not previously received genetic counselling requested an appointment following the research interview.

\section{Experience of proactive genetic counselling}

One of the reasons given by probands for refusing access to uninformed relatives was their perception that the study might stir up anxiety and a reluctance to risk causing distress. This is also the basis for the traditional reluctance of doctors to proactively inform people of their at risk genetic status. ${ }^{23}$ However, none of the relatives who had experience of being approached directly by a genetic centre and offered counselling admitted to anger or annoyance about this, and the relatives as a group were almost unanimous in the view that knowing about their genetic risk had been beneficial rather than harmful. Only one relative out of 48 said that he/she would have preferred not to be told. It is of course possible that the relatives who were not interviewed might have less positive views about being informed of their genetic risk.

\section{Acceptability and responsibility for communicating genetic risk information}

Asked their views about informing family members of their at risk status, nearly all the relatives as well as the probands (ref proband paper) said that this would be acceptable if the approach came from a member of the family. Interestingly, more relatives than probands thought it acceptable for geneticists (90\% of relatives, $78 \%$ of probands) or GPs (92\% of relatives, $68 \%$ of probands) to pass on this information.

Relatives and probands had very similar opinions about who should have the responsibility for passing on genetic risk information. Just over half of relatives and probands felt the responsibility lies with the family, but nearly a quarter of both groups saw this as the responsibility of the genetics service. More relatives than patients identified the GP as having a responsibility, just as more relatives saw it acceptable that the GP might pass on genetic information to relatives. A number of relatives as well as probands and GPs described a shared responsibility between families and health professionals.

\section{CONCLUSION}

The present study is the first to look in detail at genetic registers, comparing the views and experiences of register service users with those receiving conventional clinical genetic services. The data show that the register service had succeeded in one of its primary aims, that of extending genetic counselling to a significantly larger proportion of the at risk members in families with these four disorders than was the case for the 
traditional, more reactive, method of service provision. Furthermore, the proactive approach to informing relatives about their at risk status and the availability of genetic counselling was acceptable to almost all the family members interviewed for this study and their GPs. Only half of the probands and relatives saw the family as having responsibility for communicating genetic risk information, with the other half putting forward the view that health professionals, geneticists and GPs, should have responsibility or shared responsibility for this.

Comparison of probands referred in the conventional way by other medical practitioners to either the register or the traditional services (described in the accompanying paper) found no significant differences on any of the main outcome measures of knowledge, adjustment, and preparedness, and high levels of satisfaction among users of both types of service. However, the probands made it clear that they valued the open access and ongoing contact provided by the genetic register service, and the potential value of this long term relationship was reinforced by the comments of relatives who had not received genetic counselling regarding access to the service. Most of the relatives had some degree of awareness of the genetic implications of their family illness, and in a number of cases had not sought referral because they did not think genetic counselling, and in particular reproductive decision making, was relevant to them at the time. Ideally, genetic counselling should not be a "one off" activity; many genetic disorders have different implications for at risk subjects at different stages in life, and one of the clear benefits for family members of the long term contact inherent in the register approach is the flexibility to address these issues at the time when they are most salient to the person concerned.

\section{ACKNOWLEDGEMENTS}

We are grateful to Patricia Sloper for help and advice in the design of the study; to Wendy Waterson, Johannah Ayres, and Isobel Atkinson for secretarial assistance; and to our colleagues in the three centres, especially Helen Kingston, Jill Clayton-Smith, Nicola Crawford, Ian Ellis, Jacqueline Flynn, and Rodney Harris. We particularly acknowledge the families who participated in the study and spoke so frankly about their experiences. This work was supported by a grant from the NHS National Research and Development programme (Mother and Child Health), and the NHS Executive South East.

\section{Authors' affiliations}

L Kerzin-Storrar, C Wright, A Niindou, D Donnai, D Craufurd, Academic Group of Medical Genetics and Regional Genetic Service, Manchester, UK

P R Williamson, Department of Mathematical Sciences, University of Liverpool, Liverpool, UK

A Fryer, Department of Clinical Genetics, Alder Hey Hospital, Liverpool, UK

O Quarrell, Department of Clinical Genetics, Sheffield Children's Hospital, Sheffield, UK

\section{REFERENCES}

1 Miles MB, Huberman AM. Qualitative data analysis. London: Sage, 1984

2 Nuffield Council of Bioethics. Genetic screening. London: Nuffield Council of Bioethics, 1993.

3 Wilcke JTR. Late onset genetic disease: where ignorance is bliss, is it folly to inform relatives? BM 1998;317:744-47. 